\title{
COVID-19-Associated Mucormycosis, A New Incident in Recent Time: Is An Emerging Disease in The Near Future Impending?
}

\author{
Suman Kumar Ray ${ }^{1}$ Sukhes Mukherjee ${ }^{2}$ \\ ${ }^{1}$ Department of Applied Sciences, Indira Gandhi Technological and \\ Medical Sciences University, Arunachal Pradesh, India \\ 2 Department of Biochemistry, All India Institute of Medical Sciences, \\ Bhopal, Madhya Pradesh, India
}

\begin{abstract}
Address for correspondence Sukhes Mukherjee, PhD, Department of Biochemistry, All India Institute of Medical Sciences, Bhopal, Madhya Pradesh 462020 India (e-mail: sukhes.biochemistry@aiimsbhopal.edu.in).
\end{abstract}

Avicenna J Med 2021;11:210-216.

\begin{abstract}
Keywords

- Mucormycosis

- COVID-19

- glucocorticoid

- risk factor

- emerging disease

Mucormycosis (also known as black fungus) is caused by fungi of the Zygomycetes class and is the third most common invasive mycosis after candidiasis and aspergillosis. They colonize a large number of patients without invading them. Systemic glucocorticoids are currently used to treat severe Coronavirus disease 19 (COVID-19). In such patients, opportunistic fungal infections are a problem. Although COVID-19-related pulmonary aspergillosis is becoming more common, mucormycosis is still uncommon. Mucormycosis normally appears 10 to 14 days after being admitted to the hospital. Mucormycosis is a rare but dangerous infection that can make extreme COVID-19 worse.

Mucormycosis is more likely to occur in people who have diabetes mellitus and other risk factors. Mucormycosis is most likely exacerbated by concurrent glucocorticoid treatment. To improve outcomes, a high index of suspicion and aggressive management is required. Excessive usage of steroids, monoclonal antibodies, and broadspectrum antibiotics might cause the formation or worsen of a fungal infection.

A high index of suspicion and aggressive management are needed. In patients with COVID-19 infection, physicians should be vigilant of the likelihood of subsequent invasive fungal infections. To enhance results in pulmonary mucormycosis, early diagnosis and treatment are critical. Confirmation of the clinical form necessitates a combination of symptoms that are consistent with tissue invasion histologically. Combining various clinical data and the isolation of the fungus from clinical samples in culture is needed for the probable diagnosis of mucormycosis. The organism that causes mucormycosis is identified using macroscopic and microscopic morphological criteria, carbohydrate assimilation, and the maximum temperature at which they can expand. Mucormycosis must be treated with antifungal medication prescribed by a doctor. It may necessitate surgery in some circumstances, and it can result in the loss of the upper jaw and, in some situations, an eye.
\end{abstract}

published online

December 2, 2021
DOI https://doi.org/ 10.1055/s-0041-1735383. ISSN 2231-0770.

\footnotetext{
C 2021. Syrian American Medical Society. All rights reserved. This is an open access article published by Thieme under the terms of the Creative Commons Attribution-NonDerivative-NonCommercial-License, permitting copying and reproduction so long as the original work is given appropriate credit. Contents may not be used for commercial purposes, or adapted, remixed, transformed or built upon. (https://creativecommons.org/ licenses/by-nc-nd/4.0/) Thieme Medical and Scientific Publishers Pvt. Ltd., A-12, 2nd Floor, Sector 2, Noida-201301 UP, India
} 


\section{Introduction}

Mucormycosis is a systemic fungal infection caused by members of the Mucorales order that affects immunocompromised patients or have metabolic problems. ${ }^{1}$ Mucormycosis is a fungal infection spread across the body caused by the Zygomycetes class of the Mucorales order. Throughout this study of Mucorales infections, the word "mucormycosis" is used. Mucorales are the etiological agents of the fulminant disease known as mucormycosis, a fulminant disease with high morbidity and mortality rates that primarily affects immunocompromised patients.

Mucormycosis is a fungal infection caused by species from the Mucorales order. Coronavirus disease 19 (COVID-19), which was present at the time of readmission, may potentially have contributed to mucormycosis susceptibility. COVID-19 causes endothelialitis and microvascular thrombosis in the pulmonary and extrapulmonary vascular beds, ${ }^{2}$ which could exacerbate the angioinvasive effects of mucormycosis which, in turn, usually results in tissue infarction. ${ }^{3}$ COVID-19 can also induce immune dysregulation (for example, by causing lymphopenia). ${ }^{4}$ It can therefore predispose to an opportunistic infection, including mucormycosis, when coupled with steroid-induced immunosuppression. Patients on long-term steroid medication, for whatever cause, are vulnerable to opportunistic infections, one of which being fungal infections, such as black fungus. This increase has been related to the misuse of steroids in the treatment of COVID-19 patients, poorly controlled diabetes, and other issues.

Mucormycosis is caused by contact with mucor mold and also found in soil, air, and even human noses and mucus. It erodes facial structures, as it progresses through the respiratory tract. Even when clinically suspected, mucormycosis is a medical emergency.

Controlling diabetes and diabetic ketoacidosis is critical. Reduce the dose of steroids (if the patient is still on them) to stop them as soon as possible. If the patient is using other immune-modulating medicines, they should be stopped. India has a type 2 diabetes epidemic, and many diabetic patients are detected late in their condition, which could be contributing to the rise in cases. This is exacerbated by India's widespread habit of administering a mix of medications to individuals with even mild COVID-19.

Because poorly controlled diabetes is the primary problem, adequate glycemic control is necessary throughout COVID-19 patient treatment. Systemic steroids should only be administered in hypoxemic patients. In patients with normal oxygen saturation on room air, oral steroids are contraindicated. Blood sugar may be monitored if a systemic steroid is taken. Dexamethasone $(0.1 \mathrm{mg} / \mathrm{kg} / \mathrm{day})$ for 5 to 10 days should be the maximum dose and duration of steroid therapy. Mucorales can be avoided by wearing a universal mask and avoiding construction sites. Unlike in industrialized countries such as the United States, where cancer has emerged as the leading cause of mucormycosis, unchecked diabetes mellitus remains the leading cause of disease in developing countries. Furthermore, mucormycosis is fre- quently reported as a presenting symptom of diabetes mellitus in developing nations.

Clinicians should be familiar with the symptoms of this uncommon but deadly invasive mucormycosis to diagnose it quickly in COVID-19-positive patients receiving systemic corticosteroids during the current pandemic. ${ }^{5}$ The pandemic COVID-19 is still a major issue around the world. Even though numerous treatment options have been examined, none have been demonstrated to improve COVID-19 survival except systemic glucocorticoids. The widespread use of glucocorticoids, unfortunately, may result in secondary bacterial or fungal infections. Mucormycosis is rarely suspected or diagnosed, even though invasive pulmonary aspergillosis complicates the course of COVID-19. ${ }^{6}$

\section{Microbiology of Mucormycosis}

Mucormycosis is caused by fungi belonging to the order Mucorales, which are divided into six families: Mucoraceae, Cunninghamellaceae, Mortierellaceae, Saksenaceae, Syncephalastraceae, and Thamnidaceae. Rhizopus arrhizus (oryzae), Rhizopus microspores var. Rhizopodiformis, Rhizomucor pusillus, Cunninghmaella bertholletiae, Apophysomyces elegans, and Saksenaea vasiformis are the most common organisms. ${ }^{7,8}$ Mucorales thrive in both selective and nonselective media. The mycelial elements spread quickly, covering the entire plate in just a few days. The agents that cause mucormycosis are identified using macroscopic and microscopic morphological criteria, carbohydrate assimilation, and the maximum temperature at which they can expand. A hyaline appearance, vigorous development, light coloration on the reverse side of the plate (tanto yellow for most species), and variable degrees of coloration on the sporulating surface of the colonies are all important macroscopic features (from pure white to tan, brown, gray or even black). A microscopic view of Mucorales is shown in - Fig. 1. Mucormycosis can be classified based on the infection site, and they are a) rhinocerebral (sinus and brain) mucormycosis, ${ }^{9}$ b) pulmonary (lung) mucormycosis, ${ }^{10,11}$ c) gastrointestinal mucormycosis, d) cutaneous (skin) mucormycosis, and e) disseminated mucormycosis. In - Table 1, these major five types of mucormycosis are shown with key infections which can be occur in COVID-19 patients.
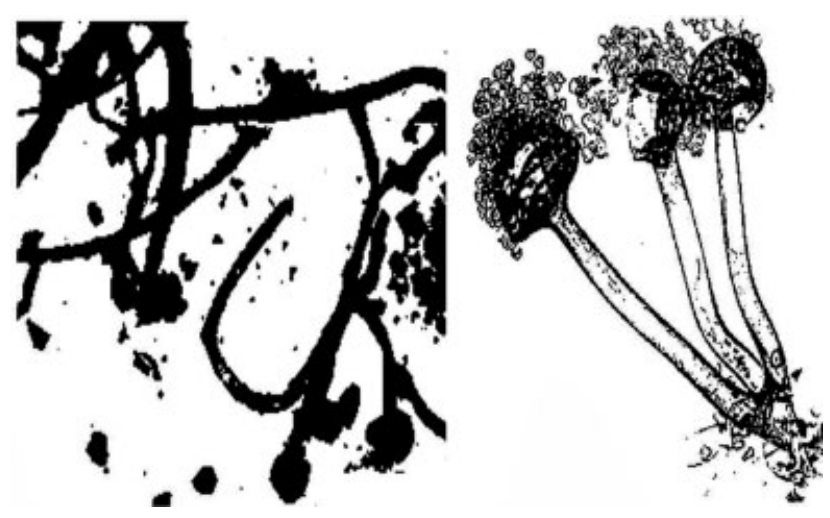

Fig. 1 Microscopic details of Mucorales. 
Table 1 Major types of mucormycosis that can infect to the COVID-19 patients

\begin{tabular}{|l|l|}
\hline Types & Infection \\
\hline muinocerebral & $\begin{array}{l}\text { - With sinus infection it has the } \\
\text { potential to spread to the brain. } \\
\text { - People with uncontrolled diabe- } \\
\text { tes and those who have under- } \\
\text { gone a kidney transplant are at } \\
\text { risk. }\end{array}$ \\
\hline $\begin{array}{l}\text { Pulmonary } \\
\text { mucormycosis }\end{array}$ & $\begin{array}{l}\text { It is the most common form of } \\
\text { mucormycosis in cancer patients } \\
\text { and those who have undergone } \\
\text { an organ transplant or stem cell } \\
\text { transplant. }\end{array}$ \\
\hline $\begin{array}{l}\text { Cutaneous } \\
\text { mucormycosis: }\end{array}$ & $\begin{array}{l}\text { Happens when fungi invade the } \\
\text { body via a skin split (for example, } \\
\text { after surgery, a burn, or other } \\
\text { type of skin trauma). }\end{array}$ \\
\hline This is the most common form of \\
mucormycosis in people who \\
don't have a compromised im- \\
mune system.
\end{tabular}

Abbreviation: Coronavirus 2019, COVID-19.

\section{Pathogenesis and Clinical Manifestations}

Mucorales enter deep tissues by inhalation of spores, percutaneous inoculation, or ingestion. ${ }^{12}$ They colonize a large number of patients, but this does not always imply invasion.

The spores are encountered by the first line of defense, mononuclear and polynuclear phagocytes, once they have entered the lungs or subcutaneous tissues. Mucorales spores are killed by the phagocytes of a healthy host, which produce oxidative metabolites and defensins (cationic peptides). The presence of elevated iron concentrations in serum is another risk factor for mucormycosis. Mucormycosis is common in patients treated with deferoxamine, possibly because Mucorales use this chelant as a siderophore to obtain more iron. If the spores manage to avoid phagocytosis, they can infiltrate vessels, by virtue of their ability to bind to endothelial cells effectively. ${ }^{13}$

Treatment of deferoxamine in patients with iron and aluminum overload has been linked to mucormycosis. However, since the advent of erythropoietin, the use of deferoxamine has decreased considerably, and as a result, this factor is becoming less common in current cases of mucormycosis. Mucormycosis most often affects the sinuses (39\%), lungs (24\%), skin (19\%), brain (9\%), gastrointestinal tract (7\%), as well as disseminated disease $(6 \%)^{14}$
The disease pattern of COVID-19 can vary from mild to life-threatening pneumonia with bacterial and fungal coinfections. ${ }^{15-17}$ These patients are vulnerable to serious opportunistic infections due to comorbidities (e.g., chronic obstructive pulmonary disease) and immunocompromised conditions (e.g., breathing, intensive care unit stay).

Oropharyngeal candidiasis, pneumocystis jiroveci pneumonia, pulmonary aspergillosis, bloodstream candida infections, and other serious opportunistic infections have been recorded in patients with COVID-19 disease. ${ }^{18,19}$ In COVID-19 illness, there are a few isolated case reports of rhino-orbital mucormycosis. ${ }^{20}$ Sen and his colleagues recently published a case series of six COVID-19 disease cases of rhino-orbital mucormycosis. One patient in this series had COVID-19 and mucormycosis at the time of admission, whereas five others developed mucormycosis while receiving COVID-19 treatment with systemic steroids. ${ }^{21}$ COVID-19 disease has a high risk of causing severe pulmonary disease and alveolointerstitial pathology. It may predispose to invasive fungal infections of the airways, such as the sinuses and lungs, on its own. ${ }^{22}$ There is also a change in innate immunity due to COVID-19-related immune dysregulation, which is characterized by a decrease in T-cells, including CD4 and CD8 cells. In the future, all physicians, including ophthalmologists, should be aware of the possibility of fungal infections such as mucormycosis developing in patients with COVID-19 illness, especially in those with comorbidities and on immunosuppressive agents.

According to autopsy investigations, Mucorales access numerous organs through all three routes, including the brain via the cerebral vascular system. ${ }^{23}$ Intravascular thrombosis and intimal hyperplasia cause fungal hyphae to develop along the internal elastic lamina and expand into the artery lumen, which gets obliterated. Cerebral infarction and hemorrhagic necrosis result from vascular blockage; however, there is no hyphal invasion of brain tissue at first. Both host innate immune cells and antifungal medications are likely hindered from accessing and acting on Mucorales in this necrotic hypoxemic environment. In advanced mucormycosis, hyphal infiltration of the necrotic parenchyma occurs as a preterminal event. Giant cells and granuloma formation indicate a relatively intact immune response, which is linked to improved results. $^{24}$ Rhinocerebral mucormycosis is characterized by vascular invasion. The brain's ischemia and infarction of the brain, including the cerebrum, cerebellum, and brainstem, are caused by intravascular thrombi. Diffuse vasculitis causes aneurysms by weakening the walls of blood vessels.

\section{Prognosis and Diagnosis}

Patients with uncontrolled diabetes mellitus ${ }^{25,26}$ who develop the rhinocerebral type $\mathrm{e}^{27,28}$ have a very low survival rate. An autopsy is commonly used to diagnose a gastrointestinal tract infection. If left untreated or unsuccessfully administered, death normally occurs within 2 weeks. Suppose the time between diagnosis and treatment lengthens, the survival rate decreases. Seventy per cent of survivors have longterm consequences, such as blindness, cranial nerve damage, and surgical disfigurement. ${ }^{29}$-Fig. 2 depicts some essential 


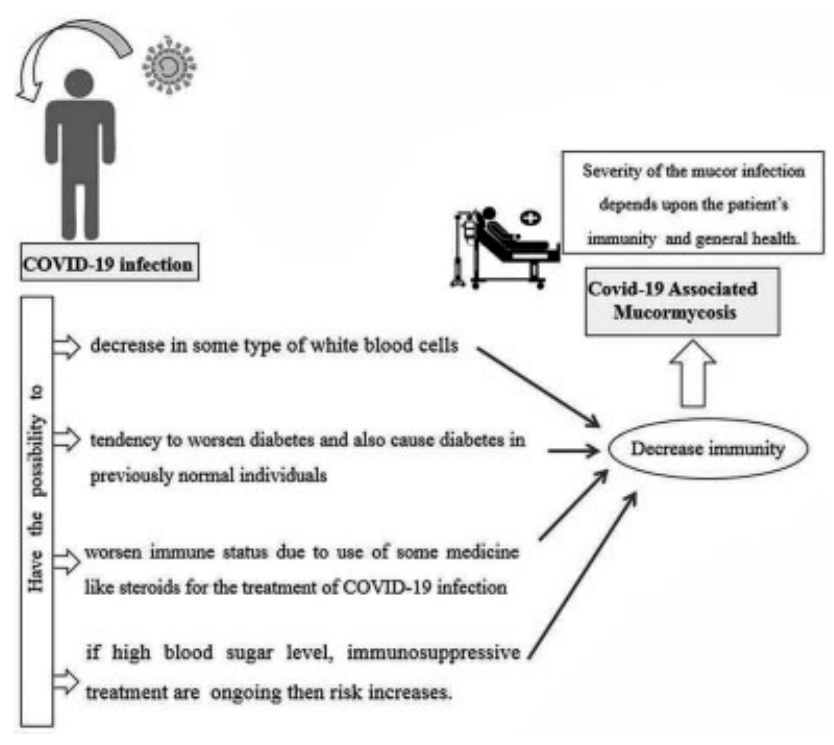

Fig. 2 Some basic factors which may play a vital role for the development of Coronavirus-19 (COVID-19)-associated mucormycosis disease.

factors that may play a role in the development of COVID-19associated Mucormycosis disease.

Early detection of mucormycosis is important, as it can improve the prognosis. ${ }^{30-32}$ According to studies, it improves survival, can minimize the need for or degree of surgical resection, and decrease disfigurement and suffering. ${ }^{33}$ Since the illness is so uncommon, having a high index of suspicion is crucial. Recognition of risk factors, evaluation of clinical symptoms, early use of imaging modalities, and timely implementation of diagnostic procedures based on histopathology, cultures, and modern molecular techniques are all part of the diagnosis process.

The sensitivity of tests that use cultures of clinical samples is limited, and there have been several reports of negative culture results, both antemortem and postmortem. ${ }^{34}$ This appears to result from aggressive specimen processing before plating, especially when the samples are biopsy specimens, which should be cut into small fragments before plating. In pulmonary types, sputum tests have sensitivity values below $25 \%$, and its specificity is uncertain, but it is widely assumed to be poor. Broad, nonseptate hyphae in culture or on slides should be viewed cautiously, because they could indicate colonization. As a result, validation of the clinical type necessitates a combination of symptoms that are consistent with tissue invasion histologically.

In an appropriate clinical setting, histological invasion of vessels by large, nonseptate hyphae branched at right angles is diagnostic. ${ }^{35}$ Combining various clinical data and the isolation of the fungus from clinical samples in culture is needed for the probable diagnosis of mucormycosis. Imaging-based diagnosis is also possible. ${ }^{36}$ Easy radiology and CT scans of rhino-orbital lesions typically reveal invasion of the sinuses, orbital displacement, and invasion of the underlying bone structures. MRI is the technique of choice when intracranial structures are affected. ${ }^{37}$ Recognition of risk factors, evaluation of clinical symptoms, early use of imaging modal-
Table 2 Major four types of diagnosis in COVID-19 associated mucormycosis

\begin{tabular}{|l|l|}
\hline Diagnosis & Basic methodology \\
\hline Clinical diagnosis & \\
\hline $\begin{array}{l}\text { Routine laboratory } \\
\text { diagnosis }\end{array}$ & Histopathology \\
\cline { 2 - 2 } & Direct microscopy \\
\cline { 2 - 2 } & Cell culture \\
\hline $\begin{array}{l}\text { Applied and emerg- } \\
\text { ing molecular } \\
\text { methods }\end{array}$ & $\begin{array}{l}\text { PCR-based procedures to detect } \\
\text { Mucorales DNA in noninvasive } \\
\text { samples, like plasma or serum. } \\
\text { Others are as follows: DNA se- } \\
\text { quencing, real-time quantitative } \\
\text { PCR }\end{array}$ \\
\hline $\begin{array}{l}\text { Noninvasive diag- } \\
\text { nostic methods }\end{array}$ & Molecular techniques \\
\cline { 2 - 2 } & Serology \\
\cline { 2 - 2 } & Metabolomics-breath test \\
\hline
\end{tabular}

Abbreviation: Coronavirus 2019, COVID-19; PCR, polymerase chain reaction.

ities, and timely implementation of diagnostic procedures ( - Table 2) based on histopathology, cultures, and modern molecular techniques are all part of the diagnosis process.

Conventional fungal culture, histopathologic examination, cytology, serology, and molecular diagnosis are the most common laboratory approaches for the diagnosis of mucormycosis. In case of immunocompromised patients, pulmonary mucormycosis, a relatively rare but severe lung fungal disease, is challenging to identify. The use of conventional cytopathology (CCP) on respiratory samples can aid in detecting Mucorales at a lower cost and in less time. ${ }^{38,39}$

Direct culturing is not suitable for quick diagnosis of mucormycosis on respiratory tract samples, since it is time-consuming and frequently yields negative results. ${ }^{40}$

\section{Treatment}

Mucormycosis can be treated after rapid diagnosis, followed by correction of predisposing factors, surgical resection, debridement, and antifungal therapy. The results of antifungal susceptibility testing trials, animal model studies, and anecdotal knowledge obtained from human cases all go into determining the best antifungal treatment. Current evidence suggests that high dose of liposomal amphotericin B is the best treatment option for this disease. ${ }^{41-45}$ In vitro, amphotericin B has demonstrated variable activity against mucormycosis-causing agents. ${ }^{46}$

Liposomal amphotericin B has lower toxicity and is welltolerated. Despite its in vitro activity against Mucorales and clinical success in a few cases, it is considered an unacceptable alternative, and there is evidence of its failure in mucormycosis patients. ${ }^{47-51}$ For the treatment of this disease, posaconazole and ravuconazole can be used. Posaconazole is more effective than itraconazole but not as effective as amphotericin B deoxycholate. It is the most effective therapeutic alternative to amphotericin $B$ at the 
moment. $^{52-54}$ Caspofungin has low activity against the agents that cause mucormycosis. Other therapeutic options are iron chelants (other than deferoxamine) and hyperbaric oxygen, which are in clinical trials. Mucormycosis can be treated with cytokines including gamma interferon and granulocyte-macrophage colony-stimulating factor. ${ }^{55,56}$

\section{Discussion}

Few recorded rhino-orbital cases associated with COVID-19 were found in a thorough analysis of the literature. Rhizopus species was the most commonly isolated species, with a mortality rate of $46 \%$. In a patient with recent-onset diabetes mellitus, a case of COVID-19 coinfection with rhino-orbital mucormycosis and ketoacidosis was identified. Reduced phagocytic activity, accessible quantities of iron due to transferrin displacement of protons in diabetic ketoacidosis, and fungal heme oxygenase, which encourages iron absorption for its metabolism, are all pathogenic mechanisms in fungal aggressiveness. Cell counts showed a progressive rise in white blood cell count and neutrophils, while lymphocytes gradually decreased in a case of extreme COVID-19 associated with fungal coinfection.

The severe acute respiratory syndrome Coronavirus (SARS-CoV-2) infection has been shown to affect CD4+ and CD8+ T-cells, which are crucial in the pathological phase of COVID-19 infection. There is a decrease in the absolute number of lymphocytes and T-cells in extreme COVID-19 cases, linked to the worst outcomes. Mucorales-specific Tcells (CD4+ and CD8+) produce cytokines that damage the fungal hyphae, such as interleukin (IL) 4, IL-10, IL-17, and interferon-gamma (IFN- $\Upsilon$ ). ${ }^{57,58}$ Lymphopenia may increase with the risk of developing invasive mucormycosis. Increasing lymphocyte count improves the adaptive immune system and induces the development of Mucorales-specific Tcells, which may help regulate the invasive infection. 59,60

Only glucocorticoids and possibly remdesivir have been shown to be effective in COVID-19. Glucocorticoids are cheap, readily available, and have been shown to minimize mortality in COVID-19 hypoxemic patients. Glucocorticoids, on the other hand, may increase the risk of secondary infections. Furthermore, the virus's immune dysregulation, and the use of immunomodulatory drugs including tocilizumab, can increase the risk of infections in COVID-19 patients. Furthermore, biomarkers for invasive aspergillosis, such as $\beta$-d-glucan and galactomannan, are not available for mucormycosis. ${ }^{61,62}$

Multiple risk factors or comorbid conditions, together with additional immunosuppression triggered by glucocorticoids, increase the net state of immune suppression in extreme COVID-19 patients, predisposing them to invasive mold infections. The index patient previously had uncontrolled blood sugar and had recently been diagnosed with end-stage kidney disease. In the presence of severe anemia, glycated hemoglobin becomes unreliable, particularly in patients undergoing hemodialysis. The positive result in this case was most likely due to improved glycemic regulation in the hospital and the prompt administration of lipo- somal amphotericin B. The optimum treatment period for mucormycosis is unknown and is determined by the clinical reaction and tolerability of the treatment regimen.

\section{Future Outlook}

Mucormycosis is becoming more common, and the case fatality rate has decreased over time. Early treatment with liposomal amphotericin B, hyperglycemia control, and surgery is essential for effective mucormycosis management. COVID19 , on the other hand, has developed a complex situation in which all three facets of management are jeopardized. To begin with, the most common treatment for extreme COVID-19, glucocorticoids, exacerbates hyperglycemia.

Diagnostic imaging and diagnosis were delayed due to the presence of acute respiratory distress syndrome and multiorgan dysfunction.

Finally, hospitals are overburdened with COVID-19 patients, and vital resources such as diagnostics and surgeries may be severely restricted. The production of mucormycosis is most likely due to glucocorticoids, implying that they should be used with caution.

Furthermore, drugs that target immune pathways, such as tocilizumab, should be avoided unless there is a strong advantage. In conclusion, physicians caring for COVID-19 patients who are seriously ill must be mindful of severe infections that can complicate the disease's path. The diagnosis of pulmonary mucormycosis necessitates a high level of clinical suspicion.

\section{Consent for Publication}

Not applicable.

\section{Funding}

None.

\section{Conflict of Interest}

The authors declare no conflict of interest.

\section{Acknowledgment}

Declared none.

\section{References}

1 Steinbrink JM Miceli MH. Mucormycosis. Infect Dis Clin North Am 2021;35(02):435-452

2 Ackermann M Verleden SE Kuehnel M et al. Pulmonary vascular endothelialitis, thrombosis, and angiogenesis in Covid-19. N Engl J Med 2020;383(02):120-128

3 Karimi-Galougahi M Arastou S Haseli S. Fulminant mucormycosis complicating coronavirus disease 2019 (COVID-19). Int Forum Allergy Rhinol 2021;11(06):1029-1030

4 Bermejo-Martin JF Almansa R Menéndez R Mendez R Kelvin DJ Torres A. Lymphopenic community acquired pneumonia as signature of severe COVID-19 infection. J Infect 2020;80(05):e23-e24

5 Seo H Kim JY Son HJ et al. Diagnostic performance of real-time polymerase chain reaction assay on blood for invasive aspergillosis and mucormycosis. Mycoses 2021. Doi: 10.1111/myc.13319

6 jrnbok . Arastehfar A, Carvalho A, van de Veerdonk FL, et al. COVID-19 associated pulmonary aspergillosis (CAPA)-from immunology to treatment. J Fungi (Basel 2020;6(2):91/jrn 
7 Ribes JA Vanover-Sams CL Baker DJ. Zygomycetes in human disease. Clin Microbiol Rev 2000;13(02):236-301

8 Bouza E Muñoz P Guinea J. Mucormycosis: an emerging disease? Clin Microbiol Infect 2006;12; (Suppl 7):7-23

9 Alekseyev K Didenko L Chaudhry B. Rhinocerebral mucormycosis and COVID-19 pneumonia. J Med Cases 2021;12(03):85-89

10 Mejia Buritica L Karduss Urueta AJ. Pulmonary Mucormycosis. N Engl J Med 2021;384(18):e69

11 Fujisaki T Inagaki J Kouroki M et al. Pulmonary actinomycosis and mucormycosis coinfection in a patient with Philadelphia chromosome-positive acute lymphoblastic leukemia undergoing chemotherapy. J Pediatr Hematol Oncol 2021. Doi: 10.1097/ MPH.0000000000002181

12 Prabhu RM Patel R. Mucormycosis and entomophthoramycosis: a review of the clinical manifestations, diagnosis and treatment. Clin Microbiol Infect 2004;10; (Suppl 1):31-47

13 Ibrahim AS Spellberg B Avanessian V. Fu Y, Edwards JE Jr. Rhizopus oryzae adheres to, is phagocytosed by, and damages endothelial cells in vitro. Infect Immun 2005;73(02): 778-783

14 Roden MM Zaoutis TE Buchanan WL, et al. Epidemiology and outcome of zygomycosis: a review of 929 reported cases. Clin Infect Dis 2005;41(05):634-653

15 Revannavar SM P S S Samaga L v K V COVID-19 triggering mucormycosis in a susceptible patient: a new phenomenon in the developing world? BMJ Case Rep 2021;14(04):e241663

16 Johnson AK Ghazarian Z Cendrowski KD Persichino JG. Pulmonary aspergillosis and mucormycosis in a patient with COVID-19. Med Mycol Case Rep 2021;32:64-67

17 Mehta S Pandey A. Rhino-orbital mucormycosis associated with COVID-19. Cureus 2020;12(09):e10726

18 Chowdhary A Tarai B Singh A Sharma A. Multidrug-resistant Candida auris infections in critically Ill coronavirus disease patients, India, April-July 2020. Emerg Infect Dis 2020;26(11): 2694-2696

19 Salehi M Ahmadikia K Badali H Khodavaisy S. Opportunistic fungal infections in the epidemic area of COVID-19: a clinical and diagnostic perspective from Iran. Mycopathologia 2020;185(04): 607-611

20 Mekonnen ZK Ashraf DC Jankowski T et al. Acute invasive rhinoorbital mucormycosis in a patient with COVID-19-associated acute respiratory distress syndrome. Ophthal Plast Reconstr Surg 2021;37(02):e40-e80

21 Sen M Lahane S Lahane TP Parekh R Honavar SG. Mucor in a viral land: a tale of two pathogens. Indian J Ophthalmol 2021;69(02): 244-252

22 Gangneux JP Bougnoux ME Dannaoui E Cornet M Zahar JR. Invasive fungal diseases during COVID-19: We should be prepared. J Mycol Med 2020;30(02):100971

23 Dando SJ Mackay-Sim A Norton R et al. Pathogens penetrating the central nervous system: infection pathways and the cellular and molecular mechanisms of invasion. Clin Microbiol Rev 2014;27 (04):691-726

24 Inglesfield S Jasiulewicz A Hopwood M et al. Robust Phagocyte Recruitment Controls the Opportunistic Fungal Pathogen Mucor circinelloides in Innate Granulomas In Vivo. MBio 2018;9(02): e02010-e02017

25 Ghaemi N Bagheri S Shirdelzade S. Pulmonary and cutaneous mucormycosis in two children with diabetes mellitus type 1 . J Pediatr Endocrinol Metab 2021;34(07):941-945

26 Miller RP Farrugia L Leask J Khalsa K Khanna N Melia L. Successful treatment of Rhizopus arrhizus rhino-orbital-cerebral mucormycosis with isavuconazole salvage therapy following extensive debridement. Med Mycol Case Rep 2021;32:39-42

27 Dallalzadeh LO Ozzello DJ Liu CY Kikkawa DO Korn BS. Secondary infection with rhino-orbital cerebral mucormycosis associated with COVID-19. Orbit 2021:1-4. Doi: 10.1080/01676830.2021. 1903044
28 Fadda GL Martino F Andreani G et al. Definition and management of invasive fungal rhinosinusitis: a single-centre retrospective study. Acta Otorhinolaryngol Ital 2021;41(01):43-50

29 Claustre J Larcher R Jouve T et al. Mucormycosis in intensive care unit: surgery is a major prognostic factor in patients with hematological malignancy. Ann Intensive Care 2020;10(01):74. Doi . Doi: 10.1186/s13613-020-00673-9

30 Wang J Li Y Luo S Zheng H. Rhinocerebral mucormycosis secondary to severe acute pancreatitis and diabetic ketoacidosis: a case report. Diagn Pathol 2021;16(01):34

31 Khatri A Chang KM Berlinrut I Wallach F. Mucormycosis after Coronavirus disease 2019 infection in a heart transplant recipient - Case report and review of literature. J Mycol Med 2021;31(02): 101125

32 Vaezi A Walther G Kurzai $O$ et al. Frequency of occurrence, seasonal variation and antifungal susceptibility of opportunistic Mucorales isolated from hospital soils in Iran. Mycoses 2021;64 (07):780-787

33 Walsh TJ Gamaletsou MN McGinnis MR Hayden RT Kontoyiannis DP. Early clinical and laboratory diagnosis of invasive pulmonary, extrapulmonary, and disseminated mucormycosis (zygomycosis). Clin Infect Dis 2012;54; (Suppl 1):S55-S60

34 Riedel S. The value of postmortem microbiology cultures. J Clin Microbiol 2014;52(04):1028-1033

35 Guarner J Brandt ME. Histopathologic diagnosis of fungal infections in the 21st century. Clin Microbiol Rev 2011;24(02): $247-280$

36 Alexander BD Lamoth F Heussel CP et al. Guidance on Imaging for Invasive Pulmonary Aspergillosis and Mucormycosis: From the Imaging Working Group for the Revision and Update of the Consensus Definitions of Fungal Disease from the EORTC/MSGERC. Clin Infect Dis 2021;72; (Suppl 2):S79-S88

37 Herrera DA Dublin AB Ormsby EL Aminpour S Howell LP. Imaging findings of rhinocerebral mucormycosis. Skull Base 2009;19(02): 117-125

38 Lackner N Posch W Lass-Flörl C. Microbiological and Molecular Diagnosis of Mucormycosis: From Old to New. Microorganisms 2021;9(07):1518

39 Jiang X Yang T Li Q et al. Liquid-Based Cytopathology Test: A Novel Method for Diagnosing Pulmonary Mucormycosis in Bronchial Brushing Samples. Front Microbiol 2018;9:2923

40 Skiada A Pavleas I Drogari-Apiranthitou M. Epidemiology and Diagnosis of Mucormycosis: An Update. J Fungi (Basel) 2020;6 (04):265

41 Hamill RJ. Amphotericin B formulations: a comparative review of efficacy and toxicity. Drugs 2013;73(09):919-934

42 Singh Y Ganesh V Kumar S et al. Coronavirus Disease-Associated Mucormycosis from a Tertiary Care Hospital in India: A Case Series. Cureus 2021;13(07):e16152

43 Singh AK Singh R Joshi SR Misra A. Mucormycosis in COVID-19: A systematic review of cases reported worldwide and in India. Diabetes Metab Syndr 2021;15(04):102146

44 Ravani SA Agrawal GA Leuva PA Modi PH Amin KD. Rise of the phoenix: Mucormycosis in COVID-19 times. Indian J Ophthalmol 2021;69(06):1563-1568

45 Raut A Huy NT. Rising incidence of mucormycosis in patients with COVID-19: another challenge for India amidst the second wave? Lancet Respir Med 2021;9(08):e77

46 Sipsas NV Gamaletsou MN Anastasopoulou A Kontoyiannis DP. Therapy of Mucormycosis. J Fungi (Basel) 2018;4(03):90 . Doi: $10.3390 /$ jof 4030090

47 Moen MD Lyseng-Williamson KA Scott LJ. Liposomal amphotericin B: a review of its use as empirical therapy in febrile neutropenia and in the treatment of invasive fungal infections. Drugs 2009;69(03):361-392

48 Ostrosky-Zeichner L Marr KA Rex JH Cohen SH. Amphotericin B: time for a new "gold standard". Clin Infect Dis 2003;37(03):415-425 
49 Miceli MH Chandrasekar P. Safety and efficacy of liposomal amphotericin B for the empirical therapy of invasive fungal infections in immunocompromised patients. Infect Drug Resist 2012;5:9-16

50 Downes KJ Fisher BT Zane NR. Administration and Dosing of Systemic Antifungal Agents in Pediatric Patients. Paediatr Drugs 2020;22(02):165-188

51 Black KE Baden LR. Fungal infections of the CNS: treatment strategies for the immunocompromised patient. CNS Drugs 2007; 21(04):293-318

52 Park SH Choi SM Lee DG et al. Intravenous itraconazole vs. amphotericin B deoxycholate for empirical antifungal therapy in patients with persistent neutropenic fever. Korean J Intern Med (Korean Assoc Intern Med 2006;21(03):165-172

53 Greer ND. Posaconazole (Noxafil): a new triazole antifungal agent. Proc Bayl Univ Med Cent 2007;20(02):188-196

54 Page AV Liles WC. Posaconazole: A new agent for the prevention and management of severe, refractory or invasive fungal infections. Can J Infect Dis Med Microbiol 2008;19(04):297-305

55 Roilides E Simitsopoulou M. Immune responses to Mucorales growth forms: Do we know everything? Virulence 2017;8(08):1489-1491
56 Spellberg B Walsh TJ Kontoyiannis DP. Edwards J Jr, Ibrahim AS. Recent advances in the management of mucormycosis: from bench to bedside. Clin Infect Dis 2009;48(12):1743-1751

57 Potenza L Vallerini D Barozzi P et al. Mucorales-specific T cells emerge in the course of invasive mucormycosis and may be used as a surrogate diagnostic marker in high-risk patients. Blood 2011;118(20):5416-5419

58 Montaño DE Voigt K. Host Immune Defense upon Fungal Infections with Mucorales: Pathogen-Immune Cell Interactions as Drivers of Inflammatory Responses. J Fungi (Basel) 2020;6(03): 173

59 Ghuman H Voelz K. Innate and Adaptive Immunity to Mucorales. J Fungi (Basel) 2017;3(03):48

60 Hassan MIA Voigt K. Pathogenicity patterns of mucormycosis: epidemiology, interaction with immune cells and virulence factors. Med Mycol 2019;57; (Suppl 2):S245-S256

61 Theel ES Doern CD. $\beta$-D-glucan testing is important for diagnosis of invasive fungal infections. J Clin Microbiol 2013;51(11): 3478-3483

62 Lamoth F. Galactomannan and 1,3- $\beta-d-G l u c a n$ Testing for the Diagnosis of Invasive Aspergillosis. J Fungi (Basel) 2016;2(03):22 